\title{
Characterization of rutile passivation layers formed on Magnéli-phase titanium oxide inert anodes
}

\section{Matthew Ryan Rowles, Nicola V. Y. Scarlett, Ian C. Madsen and Katherine McGregor}

J. Appl. Cryst. (2011). 44, 853-857

\section{IU IUCr Journals CRYSTALLOGRAPHY JOURNALS ONLINE}

Copyright (C) International Union of Crystallography

Author(s) of this paper may load this reprint on their own web site or institutional repository provided that this cover page is retained. Republication of this article or its storage in electronic databases other than as specified above is not permitted without prior permission in writing from the IUCr.

For further information see http://journals.iucr.org/services/authorrights.html 


\section{Journal of \\ Applied \\ Crystallography \\ ISSN 0021-8898}

Received 27 January 2011

Accepted 2 June 2011

C 2011 International Union of Crystallography

Printed in Singapore - all rights reserved

\section{Characterization of rutile passivation layers formed on Magnéli-phase titanium oxide inert anodes}

\author{
Matthew Ryan Rowles, ${ }^{a, b}$ * Nicola V. Y. Scarlett, ${ }^{a}$ Ian C. Madsen ${ }^{c}$ and Katherine \\ McGregor
}

\begin{abstract}
${ }^{a}$ CSIRO Process Science and Engineering, Commonwealth Science and Technology Organisation, Box 312, Clayton South, Victoria 3168, Australia, ${ }^{\mathbf{b}}$ Department of Mechanical Engineering, The University of Melbourne, Parkville, Victoria 3010, Australia, and ${ }^{\mathbf{c}} \mathrm{CSIRO}$ Light Metals National Research Flagship, Box 312, Clayton South, Victoria 3168, Australia. Correspondence e-mail: matthew.rowles@csiro.au
\end{abstract}

\begin{abstract}
An ex situ characterization study has been performed on rutile passivation layers on inert anodes used for molten salt electrochemical studies. Rutile layer thicknesses were estimated using a number of ex situ methods, including laboratory and synchrotron X-ray diffraction and optical microscopy. The only phases in the anode detected by diffraction were the Magnéli phases $\left(\mathrm{Ti}_{n} \mathrm{O}_{2 n-1}\right.$, $n=5-6)$ of the unreacted anode and rutile $\left(\mathrm{TiO}_{2}\right)$, which forms on electrolysis. These measurements validate a previously developed in situ energy-dispersive X-ray diffraction analysis technique [Scarlett, Madsen, Evans, Coelho, McGregor, Rowles, Lanyon \& Urban (2009). J. Appl. Cryst. 42, 502-512].
\end{abstract}

\section{Introduction}

Traditionally, characterization of materials involved in electrochemical investigations conducted at elevated temperatures in molten salts relies upon ex situ or post mortem methodologies. Few in situ techniques have been developed owing to the particularly challenging nature of the sample environment, which may involve, for example, molten $\mathrm{CaCl}_{2}$ at $\sim 1273 \mathrm{~K}$ (McGregor et al., 2007). To assist with in situ analyses, Scarlett et al. (2009) developed a method to analyse energy-dispersive X-ray diffraction data that allows for the nondestructive phase mapping, and subsequent phase quantification by Rietveld-based (Rietveld, 1969) quantitative phase analysis (QPA), of the internal parts of an operational electrochemical cell. Issues relating to X-ray absorption by the electrolyte were thought to have limited the accuracy of the method, and the authors concluded there was a need to compare the results with other measurements taken from sectioned electrochemical cells.

The cells used in this study simulate those which would be used for the production of titanium metal from a $\mathrm{TiO}_{2}$ cathode using a Magnéli-phase (Andersson et al., 1957) material [Ebonex (Hayfield, 2002); $\mathrm{Ti}_{n} \mathrm{O}_{2 n-1}, n=4-6$ ] as a model 'inert' anode. An Ebonex anode was used as the phase changes that occur in this material during electrolysis have been substantially characterized ex situ (McGregor et al., 2006), allowing findings made during in situ experimentation to be corroborated by ex situ data. The electrochemical cell preparation is detailed by Scarlett et al. (2009), and can be summarized as follows: The anode is immersed in a molten $\mathrm{CaCl}_{2}$ electrolyte at $\sim 1273 \mathrm{~K}$ and is eventually oxidized to nonconducting $\mathrm{TiO}_{2}$ (rutile). The electrode reactions for the process are as follows:

$$
\begin{gathered}
\text { Cathode } \mathrm{TiO}_{2}+4 \mathrm{e}^{-} \rightarrow \mathrm{Ti}+2 \mathrm{O}^{2-}, \\
\text { Anode } 2 \mathrm{O}^{2-} \rightarrow \mathrm{O}_{2}+4 \mathrm{e}^{-}, \\
\text {Overall } \mathrm{TiO}_{2} \rightarrow \mathrm{Ti}+\mathrm{O}_{2} .
\end{gathered}
$$

This study examines these cells post mortem for the purpose of determining the accuracy of the methodology developed by Scarlett et al. (2009).

\section{Experimental}

\subsection{Electrochemical cell preparation}

The preparation of the electrochemical cells has been described elsewhere (Scarlett et al., 2009); a summary is provided here.

Ebonex was obtained from Atraverda Ltd (UK) and was cut into bars of approximately $70 \times 7.5 \times 2-3 \mathrm{~mm}$. The barshaped anodes were dipped into the electrolyte to give an exposed area of about $5 \mathrm{~cm}^{2}$. Five electrolyses were conducted under an ultra-high-purity argon cover gas in a $\mathrm{CaCl}_{2}-\mathrm{CaO}$ (0.2-5.0 wt\%) electrolyte, at a temperature of approximately $1223 \mathrm{~K}$ and a current density of around $0.2 \mathrm{~A} \mathrm{~cm}^{-2}$ for times ranging from 0 to $8 \mathrm{~h}$. Alumina crucibles and a pasted/dried $\mathrm{TiO}_{2}$ cathode were used. After each electrolysis was carried out for the prescribed time, the cell was cooled and sealed with epoxy resin to minimize hydration of the $\mathrm{CaCl}_{2}$, preserving the system as accurately as possible for further analysis.

\subsection{Sample preparation}

The five electrochemical cells used in the original study were sectioned parallel to the base of the crucible, exposing 
the anode, which was then removed from the solidified calcium chloride by washing overnight in a stirred warm-water bath. The sectioned anodes were then mounted in epoxy resin and polished to $1 \mu \mathrm{m}$ for microscopy and diffraction analyses.

\subsection{Data collection}

Diffraction data were collected using a Bruker General Area Detector Diffraction System (GADDS), a Panalytical X'Pert Pro Multi Purpose Diffractometer (MPD) and the Powder Diffraction beamline (Wallwork et al., 2007) of the Australian Synchrotron. Fig. 1 shows the sample geometries. On the GADDS and the synchrotron, data were collected in fixed incident beam geometry. The samples were stationary in all instruments during the data collection.

For the GADDS measurements, an incident angle of $10^{\circ}$ and $\mathrm{Cu} K \alpha$ radiation were used. The beam was collimated with crossed Göbel mirrors (Schuster \& Göbel, 1995) and defined using a $100 \mu \mathrm{m}$ circular mask, giving a beam footprint on the sample of approximately $575 \times 100 \mu \mathrm{m}$. The beam was stepped across the width of the sample in intervals of $50 \mu \mathrm{m}$, giving $\sim 70$ diffraction patterns per sample over the range $23-$ $58^{\circ} 2 \theta$.

For the synchrotron data, the incident beam energy $(8.0013 \mathrm{keV})$ and $2 \theta$ zero point were determined from a

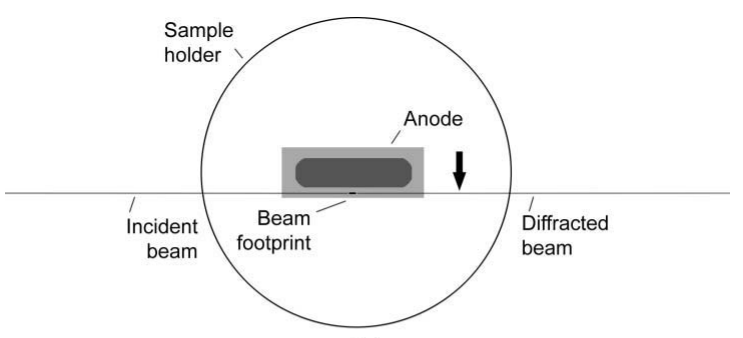

(a)

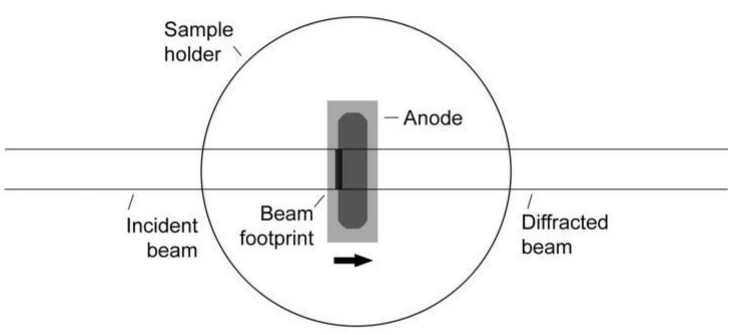

(b)

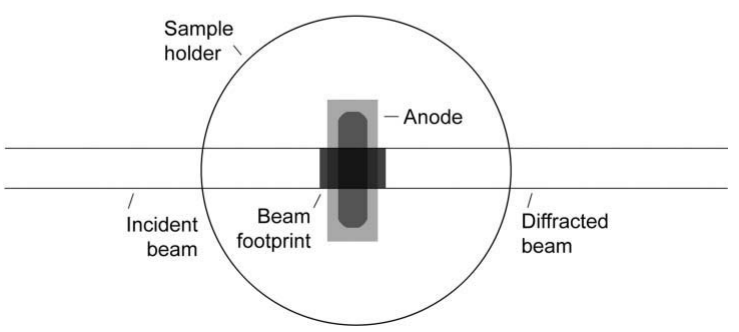

(c)

Figure 1

Schematic showing the top view of the X-ray beam/sample geometry. The arrows indicate the direction in which the sample is moved in order to scan the beam across the anode surface. (a) GADDS geometry; $(b)$ synchrotron geometry; (c) MPD geometry. standard $0.3 \mathrm{~mm}$ capillary of a mixture of NIST SRM660a $\mathrm{LaB}_{6}$ and diamond. The beam height was defined using a $0.05 \mathrm{~mm}$ slit and the incident beam angle was set at $16^{\circ}$ to give a beam footprint of $0.18 \mathrm{~mm}$. The beam width (parallel to the diffractometer axis) was defined using a $5 \mathrm{~mm}$ slit. The sample position was scanned in steps of $0.25 \mathrm{~mm}$ in the direction of the beam across the entire width of the anode, giving $\sim 18$ diffraction patterns per sample.

On the MPD, data were collected in $\theta-\theta$ symmetric diffraction geometry using nickel-filtered $\mathrm{Cu} K \alpha$ radiation. The beam width (parallel to the diffractometer axis) was defined using a $5 \mathrm{~mm}$ mask, and the incident beam footprint length was fixed at $4 \mathrm{~mm}$ using a programmable divergence slit.

The polished samples were also viewed and photographed under a binocular microscope with an integrated digital camera (Olympus SZ40; SPOT Insight Digital Camera) in reflected light mode. The magnification was calibrated using a standard graticule.

\subsection{Layer thickness determination}

The GADDS diffraction data were collated into a single surface (Fig. 2) and individual peaks were then fitted using pseudo-Voigt peaks in TOPAS (Bruker, 2009) to extract the distance versus intensity relationship, as shown in Fig. 3. In order to extract layer thicknesses, the distance/intensity profiles were fitted with hat functions, representing the rutile and Magnéli layers, and convoluted with a Gaussian function, representing beam smearing. This process removes the influence of the beam size on the measured layer thickness and allows any Magnéli/rutile phase overlap to be determined.

The synchrotron diffraction data were reduced and assembled (Rowles, 2010) into a single surface (see Fig. 4) and the layer thickness was measured by observing the difference between the first appearance of the rutile reflections and the first appearance of the Magnéli phase reflections as the sample

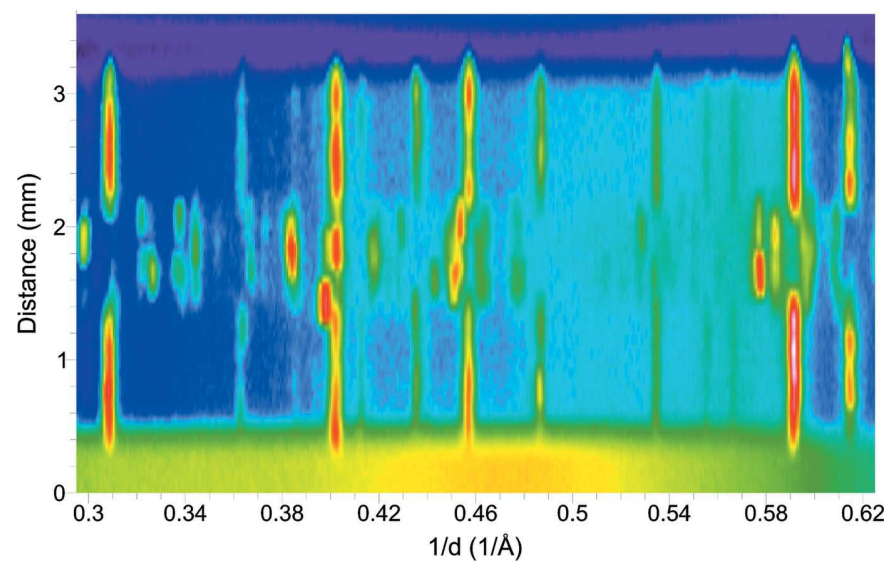

Figure 2

Accumulated GADDS diffraction patterns collected across the width of an anode operated for $240 \mathrm{~min}$ (see Fig. 1a). The data are represented as a three-dimensional plot, viewed down the intensity axis, with $1 / d$ along the $x$ axis and distance across the anode along the $y$ axis. Rutile can be seen at the outside of the anode $(0.25-1.5$ and $2.2-3.25 \mathrm{~mm})$, whilst the central section of the anode (1.5-2.2 $\mathrm{mm})$ consists of Magnéli phases. 
is scanned through the beam. The thicknesses of the layers on each side of the anode were averaged to give the calculated layer thickness for the anode. In this experiment, the size of the beam on the sample was significantly smaller than the steps between each data point on the sample surface. Owing to the coarse resolution of this technique, the layer thickness calculated gives an upper limit.

The layer thickness was calculated from the MPD data by Rietveld-based QPA as implemented in TOPAS. An example of such a fit is given in Fig. 5. The weight fractions from this analysis were converted to volume fractions and hence to a rutile layer thickness by equation (4),

$$
T_{\text {rutile }}=T_{\text {anode }} \frac{1}{2} \frac{\mathrm{wt} \%_{\mathrm{R}} / \rho_{\mathrm{R}}}{\mathrm{wt} \%_{\mathrm{R}} / \rho_{\mathrm{R}}+\mathrm{wt} \%_{\mathrm{T} 5} / \rho_{\mathrm{T} 5}+\mathrm{wt} \%_{\mathrm{T} 6} / \rho_{\mathrm{T} 6}},
$$

where wt $\%$ and $\rho$ are the weight percent and crystallographic density of rutile ( $\mathrm{R}), \mathrm{Ti}_{5} \mathrm{O}_{9}(\mathrm{~T} 5)$ and $\mathrm{Ti}_{6} \mathrm{O}_{11}$ (T6), assuming a

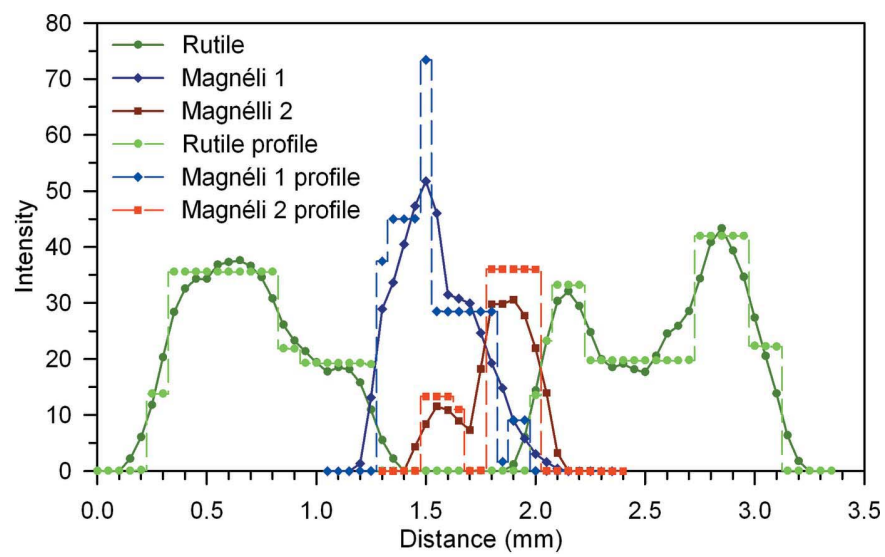

Figure 3

The measured intensity and deconvolved profile of the rutile and Magnéli peaks at $\sim 0.455 \AA^{-1}$ in the GADDS data as a function of distance across an anode operated for $240 \mathrm{~min}$, showing the layered structure. The deconvolution shows that the apparent overlap between the rutile and Magnéli phases is due to the size of the X-ray beam on the sample.

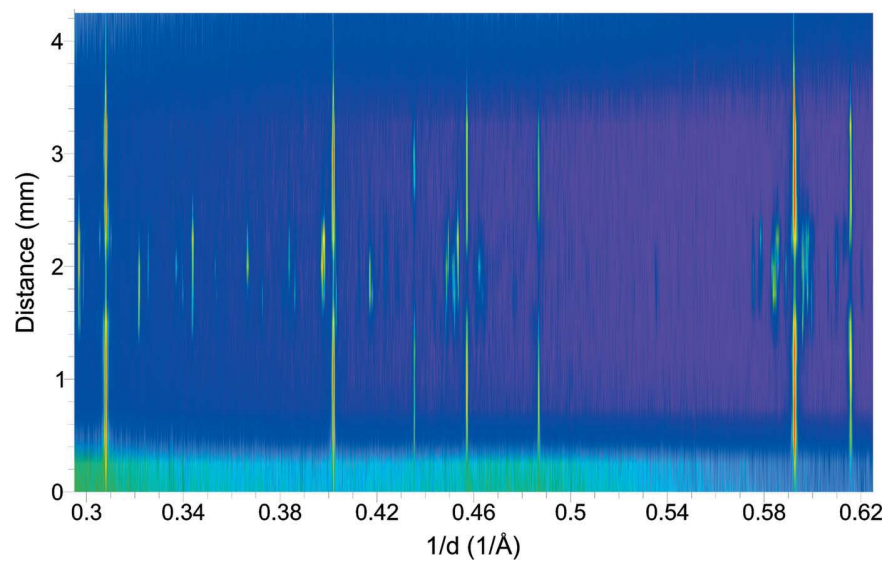

Figure 4

Accumulated synchrotron diffraction patterns collected across the width of an anode operated for $240 \mathrm{~min}$ (see Fig. 1b). The data are represented as a three-dimensional plot, viewed down the intensity axis, with $1 / d$ along the $x$ axis and distance across the anode along the $y$ axis. Rutile can be seen at the outside (0-1.5 and $2.5-4 \mathrm{~mm}$ ) of the anode, whilst the central section (1.5-2.5 mm) of the anode consists of Magnéli phases. total partitioning of the rutile and Magnéli phases. The factor of $1 / 2$ accounts for the contribution of two layers to the reported weight fraction of rutile. The structures used in this analysis were rutile (Restori et al., 1987), $\mathrm{Ti}_{5} \mathrm{O}_{9}$ (Le Page \& Strobel, 1982a) and $\mathrm{Ti}_{6} \mathrm{O}_{11}$ (Le Page \& Strobel, 1982b).

Threshold values were defined for the optical micrographs (Fig. 6) of the central region of the anode, from which diffraction data were collected, to create binary images consisting of pixels in the grey outer region and pixels in the black central core. The total number of pixels in each section was averaged over ten slices across the image and then converted to give an average layer thickness. This method was also used to give an average anode thickness to correct the fixed illumination length laboratory diffraction data.

\section{Results and discussion}

The layer thicknesses calculated from rutile and Magnéli phase appearance (GADDS and synchrotron), quantitative phase analysis (MPD), and optical microscopy are presented in Table 1 and Fig. 7. The only phases detected by diffraction are rutile and the Magnéli phases $\mathrm{Ti}_{5} \mathrm{O}_{9}$ and $\mathrm{Ti}_{6} \mathrm{O}_{11}$ (see Fig. 5). $\mathrm{Ti}_{4} \mathrm{O}_{7}$ was not detected. As it is only present at $\sim 2 \mathrm{wt} \%$, as

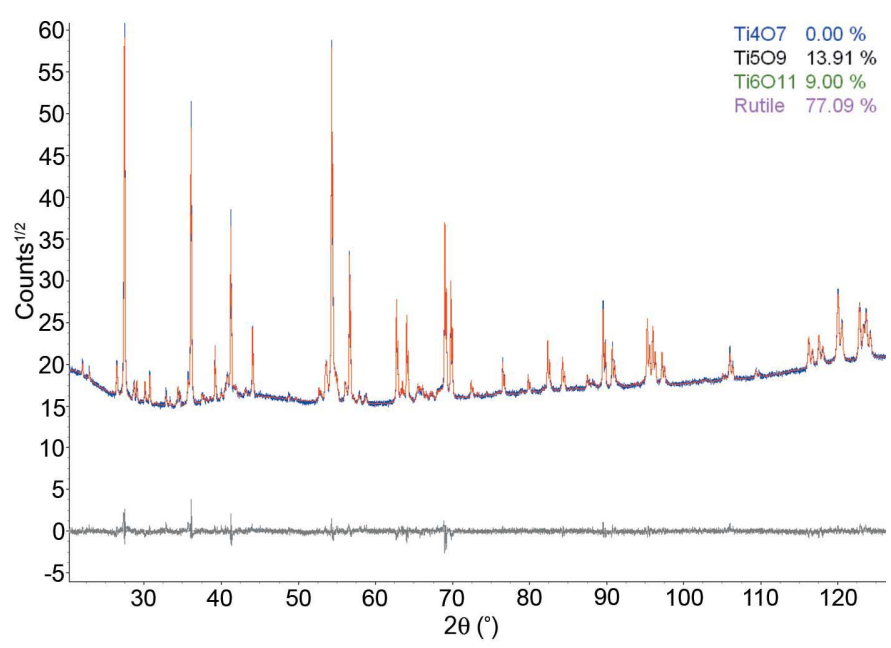

Figure 5

MPD diffraction pattern, and resultant Rietveld fit, from an anode operated for $240 \mathrm{~min}$. These diffraction data were collected in constant illumination length mode (see Fig. 1c) and show the presence of only rutile and the Magnéli phases $\mathrm{Ti}_{5} \mathrm{O}_{9}$ and $\mathrm{Ti}_{6} \mathrm{O}_{11}$.

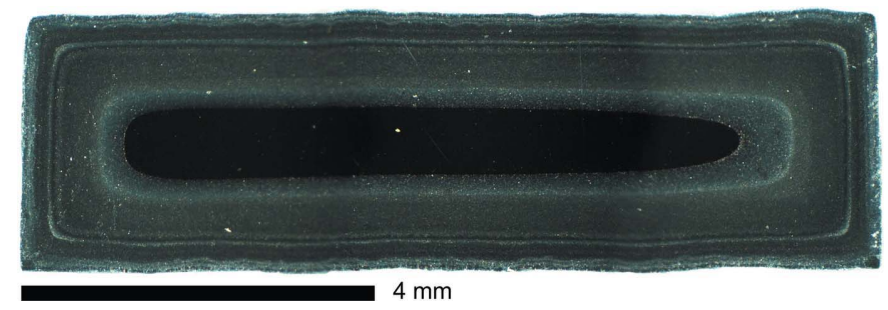

\section{Figure 6}

Optical micrograph of an anode operated for $240 \mathrm{~min}$. The cross section shows a black central core of Magnéli phases with an outer grey shell of rutile. The origin of the rutile layer colourations is attributed to variations in the electrolysis conditions during the experiment. 


\section{Table 1}

Rutile layer thickness (i) calculated by quantitative phase analysis of diffraction data collected in constant illumination length mode, (ii) measured using optical microscopy, and (iii) calculated from the appearance of the rutile and Magnéli phases when scanning an X-ray beam across the anode.

The measured thickness of the entire anode is also given. Fig. 1 shows the sample geometry for the diffraction experiments. The values given by the synchrotron data are an upper limit to the layer thickness.

\begin{tabular}{|c|c|c|c|c|c|c|}
\hline \multirow[b]{3}{*}{$\begin{array}{l}\text { Electrolysis } \\
\text { time (min) }\end{array}$} & \multicolumn{5}{|c|}{ Rutile layer thickness (mm) } & \multirow[b]{3}{*}{$\begin{array}{l}\text { Anode } \\
\text { thickness }(\mathrm{mm})\end{array}$} \\
\hline & \multicolumn{2}{|l|}{ QPA } & \multirow[b]{2}{*}{$\begin{array}{l}\text { Micro- } \\
\text { scopy }\end{array}$} & \multicolumn{2}{|c|}{ Phase appearance } & \\
\hline & Scarlett $\dagger$ & MPD & & GADDS & Synchrotron & \\
\hline 0 & $0.33(6)$ & 0.42 & $0.42(2)$ & $0.47(2)$ & 0.50 & $3.09(8)$ \\
\hline 10 & $0.38(4)$ & 0.48 & $0.51(2)$ & $0.55(7)$ & $0.63(19)$ & $3.10(5)$ \\
\hline 60 & $0.56(4)$ & 0.59 & $0.58(3)$ & $0.67(9)$ & 0.75 & $2.93(5)$ \\
\hline 120 & 0.85 & 0.82 & $0.76(2)$ & $0.84(3)$ & $1.00(18)$ & $2.94(3)$ \\
\hline 240 & $1.01(10)$ & 1.1 & $1.05(2)$ & 1.09 (4) & 1.25 & $2.90(4)$ \\
\hline
\end{tabular}

$\dagger$ From Scarlett et al. (2009). Errors have been recalculated from the original data.

determined by high-resolution synchrotron diffraction, it is quite possibly below the detection limits of the techniques utilized in this study.

The layer thicknesses calculated and measured from both the diffraction methods and optical microscopy agree very well with the thickness calculations of Scarlett et al. (2009). The slight differences between the values can be attributed to (i) variations in phase density from the theoretical and (ii) differing analysis points between this and the original study. This shows that the methodology of analysing energy-dispersive diffraction data directly with crystallographic structural information via the Rietveld method is valid.

In this work, the crystallographic densities of the phases present in the anode were used to convert the weight fraction calculated from QPA to a volume fraction and hence to a

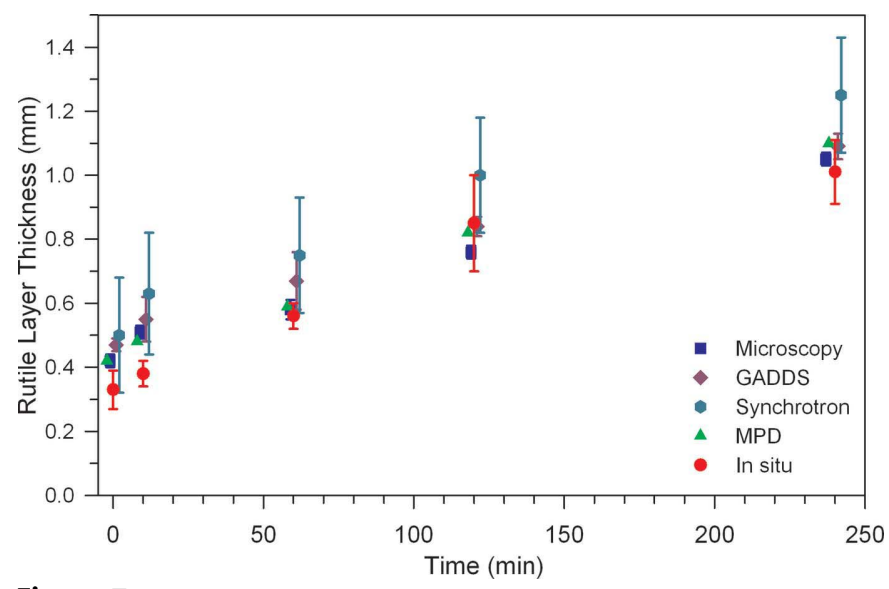

Figure 7

Rutile layer thickness (i) calculated by quantitative phase analysis of diffraction data collected in constant illumination length mode (green triangles), (ii) measured visually from optical microscopy (blue squares), and (iii) calculated from the appearance of the rutile and Magnéli phases when scanning an X-ray beam across the anode (purple diamonds and green hexagons). The in situ measurements of Scarlett et al. (2009) are given in the red circles. The values given by the synchrotron data (green hexagons) are an upper limit to the layer thickness. rutile layer thickness via equation (4). The degree of agreement between the measurements of layer thickness by phase appearance (Fig. 2 and Fig. 4), by optical microscopy (Fig. 6) and as calculated from QPA (Fig. 5) show that the assumption of crystallographic densities is valid and, therefore, the rutile layers formed are near to fully dense.

The apparent overlap in the Magnéli and rutile phases shown in the GADDS data (Fig. 3) is largely due to the size of the beam [refined to be $113(4) \mu \mathrm{m}$ ] on the sample. The deconvolved data reveal that the Magnéli and rutile phases only overlap by up to a maximum of approximately $75 \mu \mathrm{m}$, showing that the rutile forms following a reaction front moving through the anode. It follows from this that the rutile layer thicknesses calculated from equation (4), which assumes a total partitioning of the two phases, should have no significant bias in their values.

The anodes consist of a central core of Magnéli phases surrounded by an outer shell of rutile. Optical micrographs (Fig. 6) show that this rutile layer has significant sub-surface detail in the form of layers of differing contrast. Previous studies of this system (McGregor et al., 2006) and the current diffraction data have revealed only rutile in this outer layer. It is postulated that the colourations, because of their annular nature, are caused by variations in the electrolysis conditions during the initial electrochemical experimentation. The subsurface detail will be investigated further via electron microscopy.

\section{Conclusions}

These results validate the Scarlett et al. (2009) method as an appropriate means of quantitative analysis for energy-dispersive diffraction data. The layer thicknesses calculated by diffraction and microscopy agree very well, showing that the rutile formed from the oxidation of the Magnéli phases is near to fully dense and appears as a separate, distinct region; there is little to no interpenetration between the two phases. The colouration in the rutile layer has been attributed to variations in the electrolysis conditions.

The authors would like to thank Ludamilla Malishev and Cameron Davidson (CSIRO Process Science and Engineering) for preparing the samples for analysis and $\mathrm{Liz}$ Goodall (CSIRO Materials Science and Engineering) for collecting the GADDS data. Part of this research was undertaken on the Powder Diffraction beamline at the Australian Synchrotron, Victoria, Australia. This work was conducted under the auspices of the CSIRO Light Metals Flagship.

\section{References}

Andersson, S., Collen, B., Kuylenstierna, U. \& Magnéli, A. (1957). Acta Chem. Scand. 11, 1641.

Bruker (2009). TOPAS. Version 4.2. Bruker AXS Inc., Madison, Wisconsin, USA.

Hayfield, P. C. S. (2002). Development of a New Material - Monolithic $\mathrm{Ti}_{4} \mathrm{O}_{7}$ Ebonex Ceramic. Cambridge: Royal Society of Chemistry. Le Page, Y. \& Strobel, P. (1982a). J. Solid State Chem. 43, 314-319. 
Le Page, Y. \& Strobel, P. (1982b). J. Solid State Chem. 44, 273-281.

McGregor, K., Frazer, E. J., Urban, A. J., Pownceby, M. I. \& Deutscher, R. L. (2006). ECS Trans. 2, 369-380.

McGregor, K., Urban, A. J. \& Frazer, E. J. (2007). Proceedings of the 11th World Conference on Titanium, edited by M. Niinomi, S. Akiyama, M. Hagiwara, M. Ikeda \& K. Maruyama, pp. 127-130. Sendai: The Japan Institute of Metals.

Restori, R., Schwarzenbach, D. \& Schneider, J. R. (1987). Acta Cryst. B43, 251-257.

Rietveld, H. M. (1969). J. Appl. Cryst. 2, 65-71.
Rowles, M. R. (2010). Powder Diffr. 25, 297-301.

Scarlett, N. V. Y., Madsen, I. C., Evans, J. S. O., Coelho, A. A., McGregor, K., Rowles, M., Lanyon, M. R. \& Urban, A. J. (2009). J. Appl. Cryst. 42, 502-512.

Schuster, M. \& Göbel, H. (1995). J. Phys. D Appl. Phys. A, 28, 270275.

Wallwork, K. S., Kennedy, B. J. \& Wang, D. (2007). Ninth International Conference on Synchrotron Radiation Instrumentation, edited by J.-Y. Choi \& S. Rah, pp. 879-882. Daegu: American Institute of Physics. 